\title{
Dark-to-luminous properties of early type galaxies
}

\section{Nicola Napolitano*广}

Kapteyn Astron. Inst., Groningen, The Netherlands

E-mail: nicola@astro.rug.nl

In a sample of early-type galaxies we have found a correlation between the mass-to-light ratios $(M / L)$ gradients, $\nabla_{\ell} \Upsilon$, and the properties of their light distribution, in particular with their luminosity. Brightest galaxies appear the most dark-matter dominated. We discuss whether such a trend is expected in the $\Lambda \mathrm{CDM}$ framework. We conclude that the gradients in most of the brightest galaxies may fit in well with the $\Lambda \mathrm{CDM}$ predictions, but that there is also a population of fainter galaxies whose gradients are so low as to imply an unreasonably high star formation efficiency $\varepsilon_{\mathrm{SF}}>1$.

Baryons in Dark Matter Halos

5-9 October 2004

Novigrad, Croatia

\footnotetext{
* Speaker.

${ }^{\dagger}$ Receiving a grant from the EU within the 5th Framework Program (FP5) - Marie Curie Individual Fellowships
} 


\section{Introduction}

The apparent increase of $M / L$ in spiral galaxies $[4,26]$ has been the the main evidence of the presence of dark matter at galaxy scales. Since then $M / L$ ratios have been one of the prime tests of the ubiquity of the dark matter in galaxies and beyond $[2,1]$. The radial increase of $M / L$ with the radius or, in other words, the $M / L$ radial gradients is a measure of how much extra mass resides beyond the brightest regions of galaxies.

In early-type galaxies (ETGs), dynamical studies using different tracers at radii large enough to probe the (presumably) dark matter dominated regions have provided controversial results. Planetary nebulae ( $\mathrm{PNe}$ ), globular clusters (GCs), gaseous X-ray emission, gravitational lensing, and extended HI disks have so far ascertained the presence of both massive [20, 6, 12, 16, 8, 19, 17, 24] and weak $[11,21,18]$ dark haloes.

However, X-ray emission occurs mostly in giant elliptical galaxies, so these results are restricted to the brightest systems, while gravitational lensing studies have tended to be restricted to galaxies in cluster cores, and such a dense environment is somewhat atypical. On the other hand, extended HI rings (which are rare occurrences) are mostly located in S0 systems. Thus, neither method is really suitable for an unbiased survey of the properties of dark matter halos around elliptical galaxies.

The techniques remaining include integrated stellar light, PNe and GCs; there is no obvious reason for any of these to produce a particular selection effect on total mass (rather than on luminosity). We have collected 21 galaxies (16 ellipticals, 3 lenticulars and 2 of a transitional nature) for which suitable $M / L$ estimates based on these techniques were available in literature. Details of the galaxy sample can be found in [14].

\section{The $M / L$ logarithmic gradients and luminous galaxy properties}

In order to measure the radial growth of $M / L$ we defi ned the quantity

$$
\frac{R_{\mathrm{e}} \Delta \Upsilon}{\Upsilon_{*} \Delta r}=\frac{R_{\mathrm{e}}}{\Delta r}\left[\left(\frac{M_{\mathrm{d}}}{M_{*}}\right)_{\text {out }}-\left(\frac{M_{\mathrm{d}}}{M_{*}}\right)_{\text {in }}\right] \equiv \nabla_{\ell} \mathrm{r} .
$$

which is approximately equivalent to the logarithmic gradient of the $M / L$. Left expression of Eq. 2.1 can be used to determine the empirical values for the galaxy sample, once $M / L$ values are given at an inner and outer radii $\left(r_{\text {in }}, r_{\text {out }}\right)$. Middle expression of Eq. 2.1 allows to compute the predicted value for $\nabla_{\ell} \Upsilon$, once the dark $\left(M_{\mathrm{d}}(r)\right)$ and luminous $\left(M_{*}(r)\right)$ mass distributions and measurement radii are adopted.

Here we assume a constant $M / L$ for the stellar component, $\Upsilon_{*}$, which we found well constrained by the innermost $M / L$ dynamical estimates, $\Upsilon_{\text {in }}$ for our sample [14].

Following preliminary studies $[7,13]$ we checked for a correlation of $\nabla_{\ell} \Upsilon$ with galaxy luminous properties. We have found that ETGs $M / L$ gradients clearly correlate with luminosity and stellar mass (see Fig. 1): Spearman rank test gives a $99.9 \%$ confi dence for a positive correlation in both cases. In the same fi gure we also plot $\nabla_{\ell} \Upsilon$ against the mean effective surface brightness as given by HyperLeda catalogue (http://www-obs.univ-lyon1.fr/hypercat/): it is intriguing to notice that $\nabla_{\ell} \Upsilon$ get steeper below a surface brightness of about $21 \mathrm{mag} \operatorname{arcsec}^{-2}$ which is pretty consistent with the critical surface brightness, for mass discrepancy to show up, predicted by MOND ([22] and reference therein) once a $M / L=7$ typical for ETGs is taken into account. 

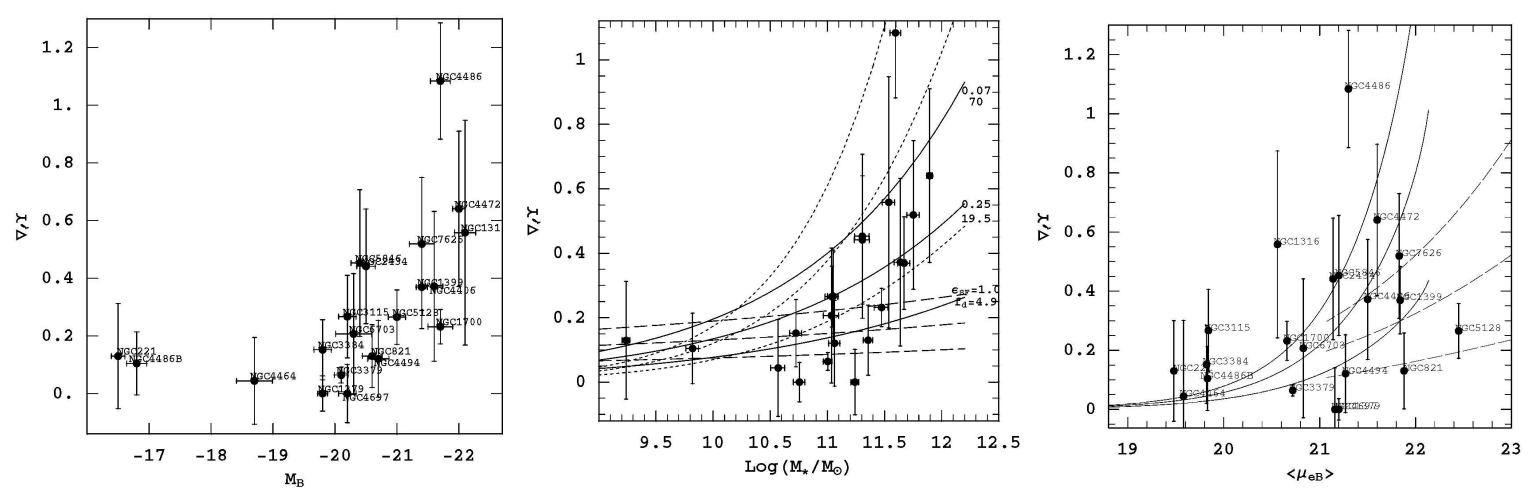

Figure 1: Empirical $M / L$ gradients versus galaxy parameters: the total luminosity $M_{\mathrm{B}}$ (left), the total stellar mass $M_{*}$ (center), the mean effective surface brightness $\left\langle\mu_{e, B}\right\rangle$ (right). Error bars are 1- $\sigma$ level. Lines are model predictions corresponding to the nominal $R_{\mathrm{e}}-M_{*}$ relation and $\pm 1 \sigma$ models, parametrised with different $\varepsilon_{\mathrm{SF}} / f_{\mathrm{d}}$ values (marked in the figure). The dashed line in the $\left\langle\mu_{e, B}>\right.$ plot show the expected model for the dwarf galaxies.

\section{Comparison with $\Lambda$ CDM expectations}

The trend of the $\nabla_{\ell} \Upsilon$ with the stellar masses (and luminosity) can be tested within the $\Lambda$ CDM framework $\left(\Omega_{\mathrm{m}}=0.3, \Omega_{\Lambda}=0.7, \sigma_{8}=0.9\right)$. We have considered spherical representations of ETGs' mass profi les, using a constant $-M / L$ model for the stellar distribution, taken as an Hernquist [9] profi le, plus a NFW model of the dark halo $[15,5]$.

Using the scaling relations which characterise both the luminous and dark matter distributions, i.e. the ETGs' size-mass relation $\left(R_{\mathrm{e}}-M_{*}[23,14]\right)$ and the $c_{\mathrm{vir}}-M_{\mathrm{vir}}$ relation as found in the $\Lambda \mathrm{CDM}$ simulations [5], both Hernquist and NFW profi les become a 1-parameter family density distributions.

This means that the model $\nabla_{\ell} \Upsilon$ can be parametrised only by the total stellar mass $\left(M_{*}\right)$ and the total dark mass within the virial radius $\left(M_{\mathrm{d}}\right)$ or equivalently by the total dark-to-luminous matter fraction $f_{\mathrm{d}}=M_{\mathrm{d}} / M_{*}$. The dark-to-luminous fraction can also easily be related to the star formation effi ciency, $\varepsilon_{\mathrm{SF}}$, assuming baryon conservation, such as $\varepsilon_{\mathrm{SF}}=4.9 / f_{\mathrm{d}}$ (see [14] for further details). In Fig. 1 the model $\nabla_{\ell} \Upsilon$ are compared to the observed gradients: data do not seem to follow the simple increase of $\nabla_{\ell} \Upsilon$ with $M_{*}$ that is expected for a "universal" $\varepsilon_{\mathrm{SF}}$, and the empirical increase appears to be sharper. This is shown in Fig. 2 where the best-fi $t \varepsilon_{S F}\left(f_{\mathrm{d}}\right)$ are plotted against the stellar masses: while the more massive (brightest) sample is confi ned within the physical meaningful $\varepsilon_{\mathrm{SF}}$ range marked by the dashed lines, less massive galaxies show mostly unphysical $\varepsilon_{\mathrm{SF}}(>1)$ values. It is statistically signifi cant the presence of a mass scale $\left[\log \left(M_{0} / M_{\odot}\right) \sim 11.2, M_{0} \sim 1.6 \times 10^{11} M_{\odot}\right]$ where this transition occurs.

In [14] we have shown that the controversy of the unphysical $\varepsilon_{\mathrm{SF}}$ for the low-mass systems can be solved if we accept that these systems resides in low-concentration dark matter haloes, inconsistent with the "concordance" model. In Fig. 2 we show how clues on the formation effi ciency (or equivalently dark-to-luminous fraction) can be affected by the cosmology: in the right panel we show the best-fit $\varepsilon_{S \mathrm{~F}}$ obtained for $\sigma_{8}=0.75$ (as recently proposed by $[10,25]$ ). The effect of smaller perturbation fluctuation has, among the others, the effect of producing a lower $c_{\mathrm{vir}}-M_{\mathrm{vir}}$ 

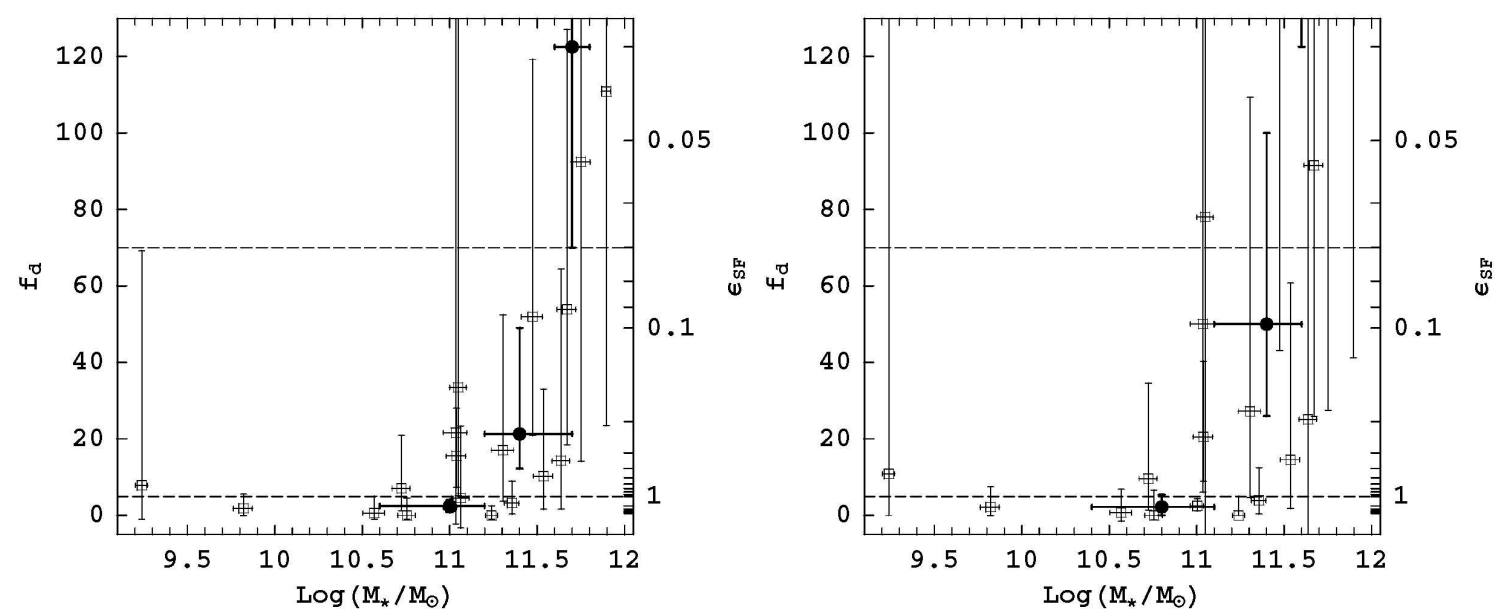

Figure 2: Efficiency of star formation versus total luminous mass, for early-type galaxy data fitted to $\Lambda \mathrm{CDM}$ models (open squares) assuming $\sigma_{8}=0.9$ (left) and $\sigma_{8}=0.75$ (right). Plausible upper and lower bounds on these parameters are shown as dotted lines. Full points are the median values from the distribution of the best-fit values. The data points of NGC 2434 and NGC 4486 are above the plot limits.

relation which partially alleviates the "exceeding stellar production" (none of the galaxies is now inconsistent with $\varepsilon_{\mathrm{SF}}=1$ ).

If we want to keep the "concordance" model, a viable mechanism to alter the dark matter distribution and then their dark-matter-only predicted $c_{\text {vir }}$ can be the classic baryon's adiabatic contraction [3]. We fi nally remark that, albeit less sensible to the cosmology, the trend of $\nabla_{\ell} \Upsilon$ with the surface brightness is well reproduced also in the $\Lambda \mathrm{CDM}$ framework and as a consequence of the existing scaling relations of both luminous and dark matter and it is not an exclusive prediction of MOND.

\section{Conclusions}

We have discussed the correlations between the $M / L$ gradients and luminous properties of early-type galaxies, in particular their luminosity, stellar mass and surface brightness. It is found that the brightest galaxies show steeper $M / L$ gradient, and that the fainter galaxies have almost null $M / L$. We have compared these observational evidence with predictions in $\Lambda \mathrm{CDM}$ cosmogony. We have considered ETGs as a two-component systems (a luminous Hernquist spheroid in a NFW dark matter profi le) and used the well known scaling relations of both luminous and dark matter distribution in order to reproduce realistic dark-to-luminous properties. The $M / L$ gradient are parametrised with the total dark-to-luminous matter fraction $f_{\mathrm{d}}=M_{\mathrm{d}} / M_{*}$ and equivalently star formation effi ciency, $\varepsilon_{S F}$.

We have shown that the joined effect of scaling relations and dark-to-luminous matter fraction, corresponding to realistic star formation efficiencies, allows to reproduce the observed $\nabla_{\ell} \Upsilon$ for stellar masses larger than $M_{0} \sim 1.6 \times 10^{11} M_{\odot}$ (corresponding to a total luminosity of about $M_{B}=$ -20.5), while less massive (luminous) systems mismatch the model prediction for physical $\varepsilon_{\mathrm{SF}}$ values. This controversy can be solved if we assume that these low luminosity galaxies reside in dark haloes less concentrated then expected from N-body simulations. 


\section{References}

[1] Bahcall N.A., Lubin L.M., Dorma V., 1995, ApJ, 447, L81

[2] Bertola F., Pizzella A., Persic M., Salucci P, 1993, ApJ, 416, L45

[3] Blumenthal, G. R., Faber, S. M., Flores, R., \& Primack, J.R., 1986, ApJ, 301, 27

[4] Bosma A., van der Kruit P.C., 1979, A\&A, 79, 281

[5] Bullock J.S., et al., 2001, MNRAS, 321, 559

[6] Buote D.A., Jeltema T.E., Canizares C.R., Garmire G.P., 2002, ApJ, 577, 183

[7] Capaccioli, M., Napolitano, N. R. \& Arnaboldi, M. 2003, Sakharov Conference of Physics, Moskow, June 2002, [preprint:astro-ph/0211323]

[8] Côté P., McLaughlin D.E., Cohen J.G., Blakeslee J.P., 2003, ApJ, 591, 850

[9] Hernquist L., 1990, ApJ, 356, 359

[10] McGaugh S.S., Barker M.K., de Blok W.J.G., 2003, ApJ, 584, 566

[11] Méndez, R.H. et al., 2001, ApJ, 563, 135

[12] Napolitano N.R., Arnaboldi M., Capaccioli M., 2002, A\&A, 383, 791

[13] Napolitano, N.R. et al. 2003, IAUS, 220, 93 [preprint astro-ph/0310798]

[14] Napolitano, N.R. et al. 2004, MNRAS, in press [preprint astro-ph/0411639]

[15] Navarro J.F., Frenk C.S., White S.D., 1997, ApJ, 490, 493

[16] Oosterloo, T. A., Morganti, R., Sadler, E. M., Vergani, D., \& Caldwell, N., 2002, AJ, 123, 729

[17] O’Sullivan, E. \& Ponman, T.J., 2004b, MNRAS, 354, 935

[18] Peng E.W., Ford H.C., Freeman K.C., 2004, ApJ, 602, 685

[19] Richtler T. et al., 2004, AJ, 127, 2094

[20] Romanowsky A.J., Kochanek C.S., 2001, ApJ, 553, 722

[21] Romanowsky A.J. et al., 2003, Science, 301, 1696

[22] Sanders, R. H. \& McGaugh, S. S. 2002, ARA\&A, 40, 263

[23] Shen S., et al., 2003, MNRAS, 343, 978

[24] Treu, T. \& Koopmans, L.V.E., 2004, ApJ, 611, 739

[25] van den Bosch F.C., Mo H.J., Yang X., 2003, MNRAS, 345, 923

[26] Whitmore, B.C., Forbes, D.A., \& Rubin, V.C., 1988, ApJ, 333, 542 